\title{
Creative Search Using Pataphysics
}

\author{
Fania Raczinski, Hongji Yang, Andrew Hugill \\ Institute of Creative Technologies \\ De Montfort University \\ Leicester LE1 9BH, UK \\ \{fania, hyang, ahu\}@dmu.ac.uk
}

\begin{abstract}
This paper looks at defining, analysing and practicing how creativity can be applied to search tools. It defines creativity with respect to search and discusses how these concepts could be applied in software engineering using principles from the pseudo-philosophy of pataphysics. The aim of the proposed tool is to generate surprising, novel, humorous and provocative search results instead of purely relevant ones, in order to inspire a more creative interaction between a user, their information need and the application. A proofof-concept prototype is described to justify the ideas presented before implications and future work are discussed.
\end{abstract}

\section{Author Keywords}

Creative computing; pataphysics; information retrieval; creative technologies.

\section{ACM Classification Keywords}

F.3.m. Logics and Meanings of Programs: Miscellaneous H.3.3. Information Search and Retrieval: Search Process J.5. Arts and Humanities: Literature

\section{General Terms}

Algorithms; Design, Experimentation.

\section{INTRODUCTION}

Imagine a web search engine that does not quite return the results you expect. For example, imagine you search for "animal" and the top three results are a list of animals in the Emperor's possession, followed by instructions about embalming animals and information on a society for animal training. Google's top search results for this query on the other hand return the webpage of an action sports lifestyle brand, the Wikipedia article and a BBC (British Broadcasting Corporation) page about animal videos. While there is certainly nothing wrong with Google's results, they are simply not very inspiring. The first example of search

Permission to make digital or hard copies of all or part of this work for personal or classroom use is granted without fee provided that copies are not made or distributed for profit or commercial advantage and that copies bear this notice and the full citation on the first page. Copyrights for components of this work owned by others than ACM must be honoured Abstracting with credit is permitted. To copy otherwise, or republish, to post on servers or to redistribute to lists, requires prior specific permission and/or a fee. Request permissions from Permissions@ acm.org.

C\&C '13, June 17 - 20 2013, Sydney, NSW, Australia Copyright 2013 ACM 978-1-4503-2150-1/13/06...\$15.00. results is adapted from Jorge Luis Borges's Chinese Encyclopaedia [4] which lists several creative definitions of the term "animal". Whilst they might not provide the kind of information we were initially seeking (if we even had a clear idea of the kind of answers we wanted), they are still perfectly valid results for the query and might even provoke a smirk upon their encounter. These are the kind of search results we are aiming for; strange, creative, surprising, inspiring and possibly funny (which some would call irrelevant) yet perfectly valid.

Pataphysics can provide some useful techniques that are very suitable for creative computing. Hendler and Hugill first suggested the use of three of its principles: clinamen, syzygy and anomaly, in their "Syzygy Surfer" [15].

"The ambiguity of experience is the hallmark of creativity, that is captured in the essence of pataphysics. Traversing the representations of this ambiguity using algorithms inspired by the syzygy, clinamen and anomaly of pataphysics, using a panalogical mechanism applied to metadata, should be able to humanize and even poeticize the experience of searching the Web. " [15]

In the rest of this paper we will introduce creativity and pataphysics and explain how they are used for our algorithms and the general philosophy during the development. We then discuss some of the implementation details for our proof-of-concept prototype and speculate on users and uses of the tool. We conclude the paper with a short discussion on further work.

\section{CREATIVITY AND PATAPHYSICS}

\section{Creativity}

We define creativity as "the ability to use original ideas to create something new and surprising of value". Here, we generally speak of creative ideas rather than products, since we believe creative products merely provide evidence of a creative process that has already taken place. Creativity is often divided into two types, one is a personal everyday type of creativity (P-creativity [2] or mini-c/little-c creativity [21]) and the other is a more eminent historical type (H-creativity [2] or Pro-c/Big-C creativity [21]). Margaret Boden further divides creativity into three categories [2, with some additional descriptions from 17, $21,22]$, the concepts of which are also described in Kaufman \& Beghetto's Four-C model [21].

- Combinational creativity: making unfamiliar combinations of familiar ideas; juxtaposition of 
dissimilar; bisociation; deconceptualisation, interpretive process of constructing and understanding

- Exploratory creativity: exploration of conceptual spaces; noticing new things in old spaces, interpretive process of constructing and understanding

- Transformative creativity: transformation of space; making new thoughts possible by altering the rules of old conceptual space, transformative learning

Boden also argues that creative ideas are surprising because they go against expectations and she believes that constraints support creativity and are even essential for it to happen. She says that constraints map out a territory of structural possibilities which can then be explored, and perhaps transformed to give another one [2]. This view supports our use of pataphysical concepts or constraints to enable creativity in search tools.

In many cases (especially of P-creative or mini-c types), both the originality and the value of a creative idea are evaluated using subjective or intrapersonal criteria [21]. Pataphysics, which represents an extreme form of subjectivity, is therefore a highly appropriate framework within which to encourage and enable creative thinking and operations.

\section{Pataphysics}

"To understand pataphysics is to fail to understand pataphysics. " [16]

Pataphysics was invented by a group of French schoolboys in France in the 1880s. One of their number was the author and playwright Alfred Jarry (1873-1907) [20], who later developed the concept both in his celebrated $U b u$ plays and in his novels and speculative writings. In short (there are over 100 equally correct definitions [6]) it can be defined as follows:

- Pataphysics is the science of imaginary solutions,

- Pataphysics is the science of the particular,

- Pataphysics is the science of the laws governing exceptions and contradictions,

- Pataphysics is to metaphysics as metaphysics is to physics, and

- Pataphysics describes a universe supplementary to this one.

We argue that pataphysics can facilitate creative computing. A pataphysical grammar can consist of exceptions, syzygies, anomalies, clinamen, antinomies, contradictions, equivalents and imaginaries. Such concepts or constraints can influence the ways in which we may navigate and transform our conceptual space. Pataphysical concepts are likely to cause surprise and could therefore be considered unconventional and provocative.
The concept of the clinamen can be understood as an unpredictable swerve which Bök called the smallest possible aberration that can make the greatest possible difference [3]. One of the most famous examples of a clinamen is Jarry's merdre (the first word in his Ubu plays). He squeezed an extra ' $r$ ' into the French word merde (meaning shit) and translates into something like pshit.

A syzygy both surprises and confuses. The concept originally comes from the field of astronomy where it denotes the alignment of three celestial bodies. In a pataphysical context it usually describes a conjunction of things, something unexpected and surprising. Unlike serendipity, a simple chance encounter, the syzygy has a more scientific purpose. A typical instance is the pun, which Jarry called the syzygy of words [20]. Next to being intentionally funny, puns demonstrate a clever use (or abuse) of grammar, syntax, pronunciation and/or semantics, often taken to a quite scientific level, such that without understanding of what is said and what the intended meaning is, the humour of the pun might be lost.

The antinomy, in a pataphysical sense, is the mutually incompatible or paradox. Mutually contradictory opposites can and do co-exist in the pataphysical universe.

\section{CREATIVE COMPUTING AND SEARCH}

\section{Creative Computing}

The concept of creative computing has existed for some time but has not yet managed to evolve into a recognised discipline within computer science. Computational creativity, on the other hand, has emerged as a field within artificial intelligence research [18] and overlaps with creative computing ideas to some extent.

It is important to differentiate between the ideas of creative computing and computational creativity. Intuitively the former is about doing computations in a creative way, while the latter is about achieving creativity through computation. You can think of the latter falling into the artificial intelligence category (using formal computational methods to mimic creativity as a human trait, see also [18]) and the former being a more poetic endeavour of how the computing itself is done, no matter what the actual purpose of the program is.

As a good example of creative computing, consider the International Obfuscated C Code Contest [19]. The competition revolves around writing compilable/runnable code, while visually appearing as obfuscated as possible. They value unusuality, obscurity and creativity but expect contestants to follow the strict rules and constraints of the $\mathrm{C}$ programming language.

Examples of computational creativity are Simon Colton's Painting Fool [9] or Harold Cohen's AARON [8]; both are computer programs that paint pictures. Kurzweil's Cybernetic Poet [23] is a classic example of a program that produces poetry. 
Our search tool can be seen from both perspectives and therefore somewhat lies in-between. We want to use creative techniques to come up with refreshing results to provide a counter-inspiration for the relevant results provided by Google or other mainstream Web search engines. We (are trying to) use creative techniques to build something that (hopefully) also has a creative purpose and value.

\section{Search}

In simple terms, a typical search process can be described as follows. A user is looking for some information so she or he types a search term or a question into the text box of a search engine. The system analyses this query and retrieves any matches from the index, which is kept up to date by a web crawler. A ranking algorithm then decides in what order to return the matching results and displays them for the user. In reality of course this process involves many more steps and levels of detail, but it provides a sufficient enough overview.

From the users' point of view the search process can be broken down into four activities [31] reminiscent of classic problem solving techniques [29]:

1. Problem identification $=$ information need $(\mathrm{IN})$,

2. Need articulation $=$ IN in natural language terms,

3. Query formulation = translate IN into query terms, and

4. Results evaluation $=$ compare against IN.

Searching can be thought of in two ways, information lookup (searching) and exploratory search (browsing) [11, 24]. A situation where an information need cannot easily be articulated or in fact is not existent (the user is not looking for anything specific) can be considered a typical case of exploratory search and describes the kind of search that is most suited to our proposed tool.

Most big search engines like Google, Baidu or Bing focus on usefulness and relevance of their results. [13, 1, 26] Google uses over 200 signals [14] that influence the ranking of web pages including their original PageRank algorithm [5]. We can only speculate whether these signals also take into account any creative factors due to their secrecy. Other search engines like YossarianLives (currently in alpha release) [32] concentrate on purely abstract concepts like metaphors for their search algorithms.

Any information retrieval process is constrained by factors like subject, context, time, cost, system and user knowledge [25]. Such constraints should be taken into consideration in the development of any search tool. A web crawler needs resources to crawl around the Web, language barriers may exist, the body of knowledge might not be suitable for all queries, the system might not be able to cater for all types of queries (e.g. multi-word queries), or the user might not be able to understand the user interface, and many more. It is therefore imperative to eliminate certain constraining factors (for example by targeting a very specific audience or filtering the amount of information gathered by a crawler from web pages).

\section{PROPOSED SYSTEM}

The general concept of the project described in this paper is pataphysical web searching and the following three points summarize its main aims:

- search the Web for suitable answers to a given query,

- return results as a list or a mixture of data structures, and

- present pataphysical results (rather than relevant ones).

\section{Principles}

The essence of the proposed search tool lies in its algorithms which make the difference to traditional search engines. The philosophical ideology behind the tool is fundamentally different. Our system will still consist of the main components typically found in Web search engines (crawler, index and ranking) but they will have slightly different inner workings and target a different audience of users.

To link back to some of the creative, pataphysical concepts we have discussed earlier, let us put some of the ideas for our tool into perspective. The constraints for our conceptual space are the pataphysical rules that we want to apply to our data. We use those rules to explore, combine and transform our space; giving us the flexibility and freedom we need to find interesting results.

We developed the idea of pataphysicalising data as the process of applying such pataphysical rules in order to produce creative search results. This pataphysicalisation process forms a central component of our system (see Figure 1) and influences all areas of the search tool.

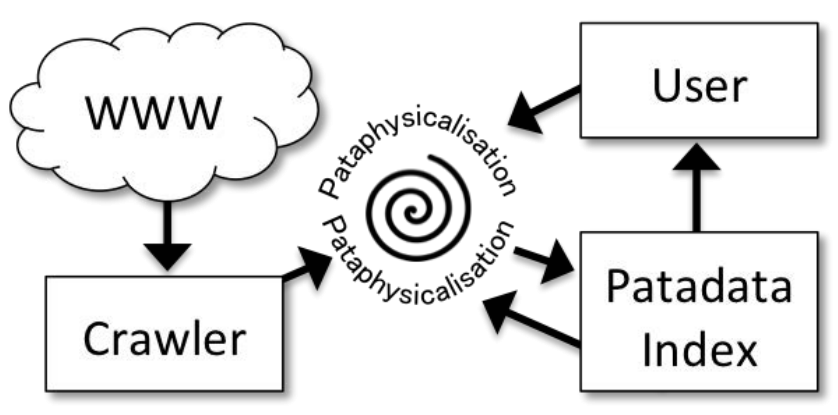

Figure 1. Pataphysicalisation as a central component

Our index will contain what Hendler and Hugill have called patadata [15]. Patadata is to metadata as metadata is to data - inspired by one of the definitions of pataphysics: that which is above that which is after physics [20]. This suggests that patadata provides another layer of information above information. If metadata helps us organise information semantically then patadata is for organising information pataphysically. If metadata is objective then patadata is subjective and that is precisely what pataphysics is for. 


\section{Prototype}

The prototype described here (see Figure 2) was developed as a proof-of-concept tool to demonstrate some example search results using pataphysical algorithms. In this case the results are limited to the text of Alfred Jarry's Exploits and Opinions of Dr. Faustroll, Pataphysician [20] and only the main algorithmic functionality of this prototype is discussed here.

\section{Pataphysical Search!}

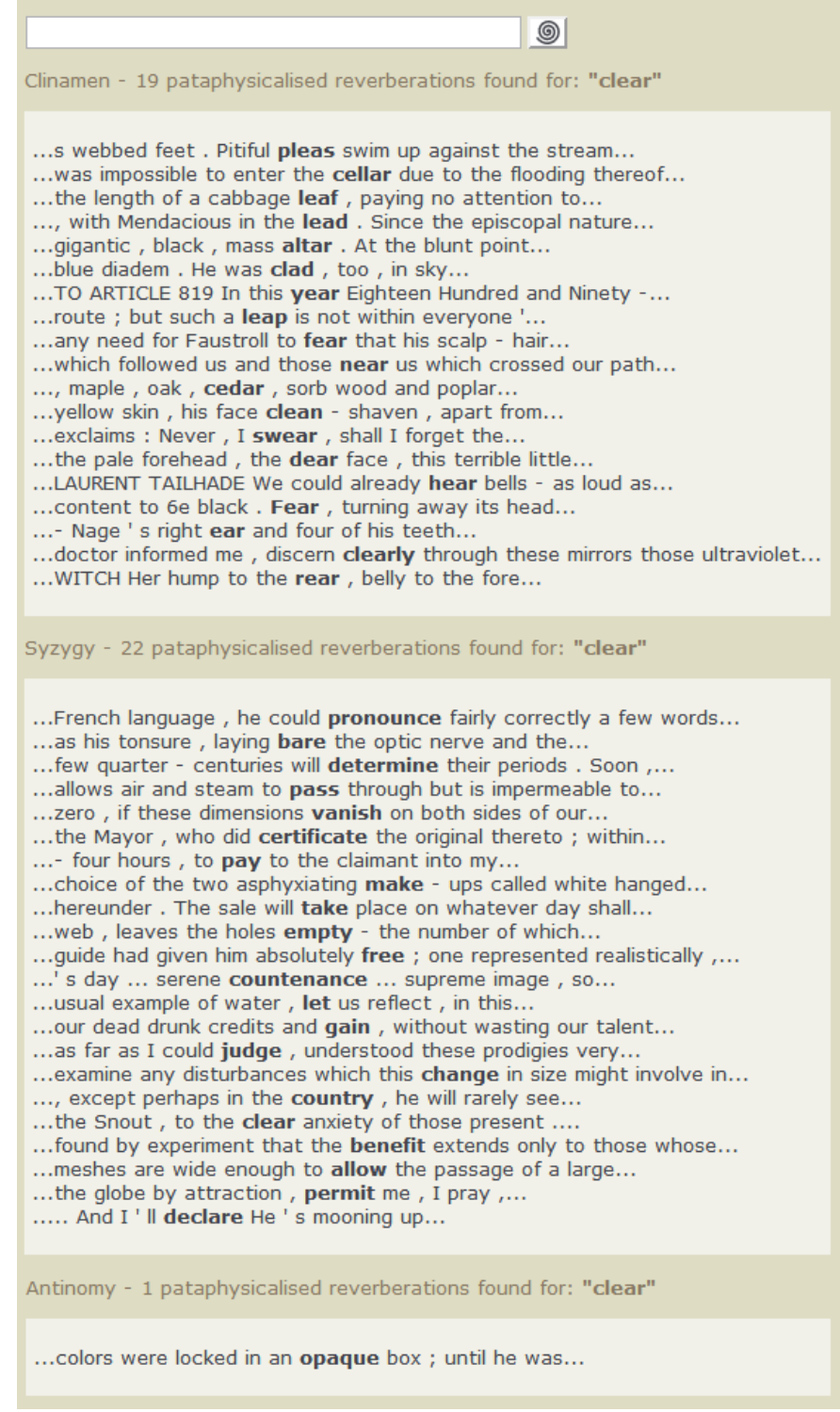

Figure 2. Screenshot of search results for the query "clear"

In short, the prototype's workflow can be described as follows:

1) tokenise text and remove stopwords to build index,

2) query triggers the three pataphysical functions,

3) each function finds matches for query as described above,
4) retrieve some words before/after match for context, and

5) return list of resulting sentences.

The three functions inspired by pataphysics (clinamen, syzygy and antinomy) are described in more detail in the next section. Figure 2 shows a screenshot of the resulting list of results for the query clear. The specific results for each of the three methods are simply a few words surrounding the pataphysicalised query term from within the book, which does not necessarily represent complete sentences but simply provides some context for the result.

The same principles and algorithms can be applied to different types of media, for example images or video and even sound. The complete tool would include a mixture of different types of media in its results with various styles of displaying them.

\section{Algorithms}

The clinamen function uses the Damerau-Levenshtein algorithm [10], which measures the distance between two strings (with 0 indicating equality), to find words that are similar but not quite the same. The distance is calculated using insertion, deletion, substitution of a single character, or transposition of two adjacent characters. We are basically asking the program to return matches $(v)$ that are of distance two or one to query term $t$, meaning they have two or one spelling errors in them (see Equation 1). While we only return matches that actually appear in the book (i.e. they exist in the index), and by doing so eliminate the introduction of new words like Jarry's merdre, the swerve or aberration is still evident.

clinamen $(t)=\{v: 0<$ dameraulevenshtein $(t, v) \leq 2\}$, for $v \in V$

For the syzygy function, we made use of the WordNet lexical database [30] using the NLTK python library [27] to find suitable results. Specifically, as shown in Equation 2, the algorithm fetches the set of synonyms (synsets) for query term $t$ first and then finds any hyponyms, hypernyms or holonyms for each of those (each of which denotes a sort of relationship or membership with its parent synonym). We then return a list of all of those related terms if they appear in the original vocabulary of the text (index $V$ ). This approach mimics the syzygy alignment of three words in a line mentioned earlier (query $\rightarrow$ synonym $\rightarrow$ hypo/hyper/holonym).

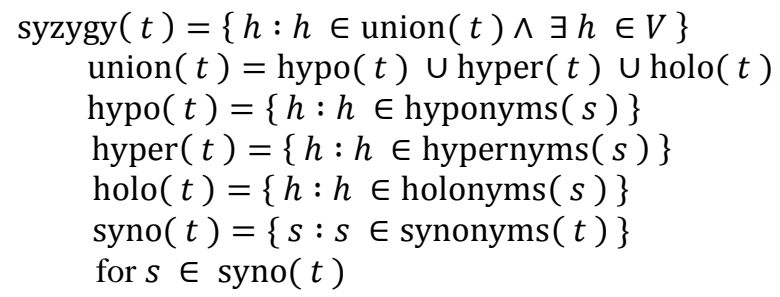

For the antinomy function we simply made use of WordNet's antonyms (opposites) (see Equation 3). We first get all synonyms for query term $t$, find any antonyms for 
those and return any that also appear in the index $V$. Naturally, not all words have an opposite, especially given WordNet's limited vocabulary of around 150,000 words, but a pataphysical antinomy should still be able to find a match. This is a big shortcoming of our prototype at this point. A better thesaurus or a larger index (e.g. based on more than one book - or, of course, the Web) could improve this function drastically.

$$
\begin{aligned}
& \operatorname{antinomy}(t)=\{h: h \in \operatorname{anto}(t) \text { and } \exists h \in V\} \\
& \operatorname{anto}(t)=\{h: h \in \operatorname{antonyms}(s)\} \\
& \operatorname{syno}(t)=\{s: s \in \operatorname{synonyms}(t)\} \\
& \text { for } s \in \operatorname{syno}(t)
\end{aligned}
$$

Table 1 shows some example matches produced by the three algorithms described above. While the syzygy and antinomy methods both work in a semantic manner, the clinamen function is purely syntactical, which becomes very obvious when seeing the different results side by side as in the table. Relying on WordNet's limited vocabulary means less matches can be found mostly because the text of Faustroll uses a very specific language and not always matches that found in the thesaurus. On the other hand, it illustrates the breadth of vocabulary used by Jarry in his writing nicely, as only those results are returned that actually appear in the book.

\begin{tabular}{|c|c|c|c|}
\hline clear & $\begin{array}{c}\text { clinamen } \\
\text { pleas, cellar }\end{array}$ & $\begin{array}{c}\text { syzygy } \\
\text { vanish, allow, } \\
\text { bare, pronounce }\end{array}$ & opaque \\
\hline solid & $\begin{array}{c}\text { sound, valid, } \\
\text { solar, slide }\end{array}$ & $\begin{array}{c}\text { block, form, } \\
\text { matter, crystal, } \\
\text { powder }\end{array}$ & $\begin{array}{c}\text { liquid, } \\
\text { hollow }\end{array}$ \\
\hline books & $\begin{array}{c}\text { boot, bones, } \\
\text { hooks, } \\
\text { rocks, banks }\end{array}$ & $\begin{array}{c}\text { dialogue, } \\
\text { authority, } \\
\text { record, fact }\end{array}$ & - \\
\hline troll & $\begin{array}{c}\text { grill, role, } \\
\text { tell }\end{array}$ & $\begin{array}{c}\text { wheel, roll, } \\
\text { mouth, speak }\end{array}$ & - \\
\hline live & $\begin{array}{c}\text { love, lies, } \\
\text { river, wave, } \\
\text { size, bite }\end{array}$ & $\begin{array}{c}\text { breathe, people, } \\
\text { domicile, taste, } \\
\text { see, be }\end{array}$ & recorded, \\
dead
\end{tabular}

Table 1. Example search results. Queries are shown in column one, algorithms used in row one.

\section{POSSIBLE APPLICATIONS}

In this section we consider the possible uses and applications for the proposed creative search tool.

Our target audience is not quite as broad as that of a general search engine like Google. Instead, we aim to specifically cater for users who can appreciate creativity or users in need of creative inspiration. Users should generally be educated about the purpose of the search tool so they are not discouraged by what might appear to be nonsensical results. Potential users could include artists, writers or poets and anybody who is looking for out-of-the-box inspirations or a refreshingly different search engine to the norm.

\section{Uses}

There are many ways a pataphysical search tool could be used across disciplines.

In literature, for example, it could be used to write or generate poetry, practically or as a simple aid for inspiration. We are not limited to poetry either; novels, librettos or plays could benefit from such pataphysicalised inspirations. One can imagine tools using this technology that let you explore books in a different ordering of sentences (a sort of pataphysical journey of paragraph hopping), tools that re-write poems or mix and match them together. Even our simple prototype shows potential in this area and could be even more powerful if we extended it to include more source texts, for example the whole set of books contained in Faustroll's library ([20] and also [12]). A richer body of texts (by different authors) would also produce a much larger index which would then possibly find many more matches through WordNet and end in a more varied list of results.

From a computer science perspective this pataphysical approach could be added to the many algorithms used by traditional search engines for purposes like query feedback or expansion (e.g. "did you mean ... "or "you might also be interested in ..." ). Depending on how creative we want the search engine to be, the higher we would rank the importance of this particular algorithm. One of the concepts related to the search tool, namely patadata, could have an impact on the development of the Semantic Web. Just as the Semantic Web is about organizing information semantically through objective metadata, patadata could be used to organize information pataphysically in a subjective way.

Our prototype tool is already being used in the creation of an online opera, provisionally entitled from [place] to [place], created in collaboration with The Opera Group ${ }^{1}$, an award-winning, nationally and internationally renowned opera company, specialising in commissioning and producing new operas. In particular, it is being used to create the libretto for one of the virtual islands whose navigation provides the central storyline for the opera. The opera will premiere in 2013 , and will continue to develop thereafter, deploying new versions of the tool as they appear.

\section{Evaluation}

Evaluating creative software is not an easy task and there are no standard approaches. Pease and Colton [28] divide it into two notions:

\footnotetext{
${ }^{1}$ www.theoperagroup.co.uk
} 
- whether an idea or artefact is valuable or not, and

- whether a system is acting creatively or not.

Following this approach, we would need to investigate each individual search result in terms of its value and creativity. This could be done by user ratings or satisfaction questionnaires. Rather than measuring the success of individual results though, we could also look at evaluation them as one set instead, similar to the blind side-by-side comparisons by the Bing search engine or the selective omission of a certain number of results by search engine MillionShort ${ }^{2}$.

The way we display and label results produced by the tool can influence how the user perceives them. Our current prototype for example separates the results into its three components but we could have equally just mixed them all together. It not always clear how each result connects to the initial query, even if we identify through which algorithm a result has been obtained. These keywords (syzygy, clinamen and antinomy) might not be helpful to users unfamiliar with the concept of pataphysics anyway and might therefore appear rather nonsensical. Whilst there is a clear logic to each search result, they might appear anomalous to the user's expectations if he received these results without knowing the philosophy of the search tool. The results could possibly appear random then, and could therefore likely to be detrimental to the user. The level of interaction between the user and the system and the feedback the tool gives to the user on its internal processes will have a large influence on the overall effectiveness, perception and appreciation of the tool.

The less obvious the processes in the background are for the user, the more difficult it might be to appreciate the search results. On the other hand, too much transparency could spoil much of the experience. After all, explaining a joke kills it. The issue therefore becomes a question of finding just the right level of transparency to satisfy curious users while at the same time not spoiling the seemingly serendipitous experience of others.

\section{FURTHER WORK AND CONCLUSION}

We are just beginning to understand the potential of such a creative search tool and its implications. There is much research left to be done, specifically in developing more and different kinds of search algorithms and evaluating the results we obtain. We could try to implement different algorithms or different pataphysical concepts within our existing prototype or built a different system altogether. We could also try to implement a fully functioning Web search engine using the algorithms described in this paper and then compare the two different types of results. It could be interesting to investigate how users perceive and use search

\footnotetext{
${ }^{2}$ www.bingiton.com and www.millionshortiton.com
}

results produced in either the book based search or an open Web based search.

Before we go into further development and programming though, it might be worth studying, evaluating and interpreting the results produced by the prototype presented in this paper. An evaluation framework for pataphysical search results is under development. A study of user's reactions to the prototype could be very interesting as well and will be part of future work in this project.

Finally, to summarise, in this paper we have introduced a new approach for a creative search tool that uses pataphysics as an underlying philosophy. We have explained how pataphysics can be applied to search algorithms in order to produce interesting results with a humorous twist. Our initial experiments within a limited domain have shown that the generated results could indeed be interpreted as being novel, surprising and useful. We have also briefly discussed ideas for applications of the tool and issues that may trigger possible further research in in the field of creative computing. We have also presented some thoughts on evaluation of our tool and future work.

\section{ACKNOWLEDGMENT}

Jim Hendler at the Rennselaer Polytechnic Institute has provided valuable advice for this project.

\section{REFERENCES}

1. Baidu The Baidu Story [online] available at http://ir.baidu.com/phoenix.zhtml?c=188488\&p=irolhomeprofile [Accessed 12 May 2013]

2. Boden, M.A. The Creative Mind: Myths and Mechanisms, London: Routledge, 2003.

3. Bök, C. 'Pataphysics: The Poetics of an Imaginary Science, Evanston, Illinois: Northwestern University Press, 2002.

4. Borges, J.L. The Analytical Language of John Wilkins in Selected Non-Fictions, Weinberger, E. (Ed.), London: Penguin Books, 2000.

5. Brin, S.and Page, L. The anatomy of a large-scale hypertextual Web search engine in Computer Networks and ISDN Systems, 30(1-7), 1998, pp.107-117.

6. Brotchie, A., Chapman, A., Foulc, T. and Jackson, K. (Eds.), 'Pataphysics: Definitions and Citations, London Institute of 'Pataphysics, London: AtlasPress, 2003.

7. Brotchie, A. Alfred Jarry: A Pataphysical Life, Cambridge, MA: MIT Press, 2011.

8. Cohen, H. AARON [online] available at http://www.kurzweilcyberart.com/aaron/history.html [Accessed 12 May 2013]

9. Colton, S. The Painting Fool [online] available at http://www.thepaintingfool.com/ [Accessed 12 May 2013]

10. Damerau, F.J. A Technique for Computer Detection and Correction of Spelling Errors in Communications of the ACM, 7(3), 1964, pp.171-176. 
11. de Vries, E. Browsing vs Searching, OCTO report 93/02, 1993.

12. Fisher, B. The Pataphysician's Library, Liverpool University Press, 2000.

13. Google Company Overview [online] available at https://www.google.com/intl/en/about/company/ [Accessed 12 May 2013]

14. Google Ten things we know to be true [online] available at https://www.google.com/intl/en/about/company/philos ophy/ [Accessed 12 May 2013]

15. Hendler, J. and Hugill, A. The Syzygy Surfer : Creative Technology for the World Wide Web, in Proc. WebSci 2011, ACM Press (2011).

16. Hugill, A. 'Pataphysics: A Useless Guide, Cambridge, MA: MIT Press, 2012.

17. Indurkhya, B. Computers and Creativity, Unpublished Manuscript, Based on the key note speech On Modeling Mechanisms of Creativity, delivered at Mind II: Computational Models of Creative Cognition, Dublin, Ireland, September 15-17, 1997.

18. International Conference of Computational Creativity [online] available at http://www.computationalcreativity.net/icce2013/ [Accessed 12 May 2013]

19. International Obfuscated C Code Contest [online] available at http://www.ioccc.org/ [Accessed 12 May 2013]

20. Jarry, A. Exploits and Opinions of Dr Faustroll, Pataphysician, Cambridge, MA: Exact Change, 1997.

21. Kaufman, J. C., \& Beghetto, R. A. (2009). Beyond big and little: The four c model of creativity. Review of General Psychology, 13(1), 1-12.
22. Koestler, A. The Act of Creation, London: Penguin Books, 1964.

23. Kurzweil, R. Cybernetic Poet [online] available at http://www.kurzweilcyberart.com/poetry/rkcp_overvie w.php [Accessed 12 May 2013]

24. Marchionini, G. From finding to understanding, Communications of the ACM, vol. 49, no. 4, pp. 41-46, 2006.

25. Marchionini, G. and Shneiderman, B. Finding facts vs. browsing knowledge in hypertext systems, Computer, vol. 21, no. 1, pp. 70-80, 1988.

26. Microsoft Bing Fact Sheet October 2012[online] available at http://www.microsoft.com/enus/news/download/presskits/bing/docs/MSBingAllUpFS.docx [Accessed 12 May 2013]

27. NLTK Project, 2012, Natural Language Toolkit [online] available at http://nltk.org/ [Accessed 12 May 2013].

28. Pease, A. and Colton, S. On impact and evaluation in Computational Creativity: A discussion of the Turing Test and an alternative proposal in Proc. AISB $2011^{\circ}$ Symposium on AI and Philosophy.

29. Polya, G. How To Solve It, 2nd ed. Princeton, New Jersey: Princeton University Press, 1957.

30. Princeton University, 2010, About WordNet [online] available at http://wordnet.princeton.edu [Accessed 12 May 2013]

31. Sutcliffe, A. and Ennis, M, Towards a cognitive theory of information retrieval, Interacting with Computers, vol. 10, pp. 321-351, 1998.

32. YossarianLives The Metaphorical Search Engine [online] available at http://www.yossarianlives.com/ [Accessed 12 May 2013] 